This item was submitted to Loughborough's Research Repository by the author.

Items in Figshare are protected by copyright, with all rights reserved, unless otherwise indicated.

\title{
State and parameter estimation of the AquaCrop model for winter wheat using sensitivity informed particle filter
}

PLEASE CITE THE PUBLISHED VERSION

https://doi.org/10.1016/j.compag.2020.105909

\section{PUBLISHER}

Elsevier

VERSION

VoR (Version of Record)

\section{PUBLISHER STATEMENT}

This is an Open Access Article. It is published by Elsevier under the Creative Commons Attribution 4.0 Unported Licence (CC BY). Full details of this licence are available at: http://creativecommons.org/licenses/by/4.0/

\section{LICENCE}

CC BY 4.0

\section{REPOSITORY RECORD}

Zhang, Tianxiang, Jinya Su, Cunjia Liu, and Wen-Hua Chen. 2020. "State and Parameter Estimation of the Aquacrop Model for Winter Wheat Using Sensitivity Informed Particle Filter". Loughborough University. https://hdl.handle.net/2134/13347176.v1. 
Original papers

\title{
State and parameter estimation of the AquaCrop model for winter wheat using sensitivity informed particle filter
}

\author{
Tianxiang Zhang ${ }^{\mathrm{a}, \mathrm{b}}$, Jinya Su ${ }^{\mathrm{c}, *}$, Cunjia Liu ${ }^{\mathrm{b}, *}$, Wen-Hua Chen ${ }^{\mathrm{b}}$ \\ ${ }^{a}$ School of Automation and Electrical Engineering, University of Science and Technology Beijing, Beijing 100083, China \\ ${ }^{\mathrm{b}}$ Department of Aeronautical and Automotive Engineering, Loughborough University, Loughborough LE11 3TU, UK \\ ${ }^{\mathrm{c}}$ School of Computer Science and Electronic Engineering, University of Essex, Colchester CO4 3SO, UK
}

\section{A R T I C L E I N F O}

\section{Keywords:}

Particle filter

Sensitivity analysis

Machine learning

Multispectral image

Unmanned Aerial Vehicle

\begin{abstract}
A B S T R A C T
Crop models play a paramount role in providing quantitative information on crop growth and field management. However, its prediction performance degrades significantly in the presence of unknown, uncertain parameters and noisy measurements. Consequently, simultaneous state and parameter estimation (SSPE) for crop model is required to maximize its potentials. This work aims to develop an integrated dynamic SSPE framework for the AquaCrop model by leveraging constrained particle filter, crop sensitivity analysis and UAV remote sensing. Both Monte Carlo simulation and one winter wheat experimental case study are performed to validate the proposed framework. It is shown that: (i) the proposed framework with state/parameter bound and parameter sensitivity information outperforms conventional particle filter and constrained particle filter in both state and parameter estimation in Monte Carlo simulations; (ii) in real-world experiment, the proposed approach achieves the smallest root mean squared error for canopy cover estimation among the three algorithms by using day forwardchaining validation method.
\end{abstract}

\section{Introduction}

Crop simulation models, providing quantitative crop growth information during the crop life-cycle, play a paramount role in sustainable agriculture management. It contributes to intelligent irrigation, nutrient management, and yield prediction before harvest, which directly promote agriculture sustainability and food security (Zhang et al., 2019). However, the prediction performance of crop model degrades significantly in real-life applications due to the presence of unknown and uncertain system parameters. In this regard, a timely and reliable Simultaneous State and Parameter Estimation (SSPE) for crop model is highly desirable to realize its full potentials. Recently, the integration of crop models and remote sensing information is drawing ever-increasing research interest in precision agriculture, where the problem is usually addressed by using various data assimilation techniques (optimization approaches at large) (Jin et al., 2018).

Crop models are able to quantitatively simulate crop physiological process at a daily basis. Due to their practical usability, a number of crop models have been developed recently from different principles such as WOFOST, DASSAT, STICS and AquaCrop model. Unlike other crop models (i.e. light driven or carbon driven), AquaCrop model is water- driven model, which is simple, robust and accurate (Steduto et al., 2009). This model has been widely applied in precision agriculture practices such as crop monitoring, intelligent irrigation management and yield prediction before harvest (Foster et al., 2017). Therefore, the AquaCrop crop model is adopted to demonstrate the proposed SSPE framework in this study.

Regarding remote sensing information, images of various spatial/ spectral resolutions can be captured by sensing platforms such as satellite, manned-aircraft and Unmanned Aerial Vehicles (UAV) (Zhang et al., 2019). Among them, UAV remote sensing is drawing increasing research interests and has become an important supplement to conventional platforms (Su et al., 2020). This is mainly due to its attractive characteristics including a relatively affordable cost, a high spatial and user-defined temporal resolution, and a good flexibility (Bansod et al., 2017). It has also been widely applied in a large number of applications such as crop stress monitoring (e.g. disease, weed, drought), crop state estimation (e.g. canopy cover, biomass, leaf area index) and crop parameter inference (Jin et al., 2018; Su et al., 2019).

In this study, UAV remote sensing is to derive canopy cover (CC) of the AquaCrop model. CC is defined as the ratio of plant leaves projected to the horizontal surface to the total ground area (Fiala et al., 2006) and

\footnotetext{
* Corresponding authors.

E-mail addresses: T.Zhang@lboro.ac.uk (T. Zhang), j.su@essex.ac.uk (J. Su), C.Liu5@lboro.ac.uk (C. Liu), W.Chen@lboro.ac.uk (W.-H. Chen).
} 
is one of the most important state variables in the AquaCrop model. The calculation of CC value is formulated as an image classification problem, which is addressed by the random forest classifier. Image pixels are segmented into wheat and non-wheat pixels, based on which the proportion of wheat pixels is calculated as the CC value. It is shown in Hamuda et al. (2016) that this machine learning based approach outperforms threshold based approaches (Su et al., 2020) and is therefore adopted in this study.

In addition, state estimation problem can be found in many applications such as crop state estimation, hazardous target tracking, hydrological parameter inference (Jiang et al., 2014; Hutchinson et al., 2019). This problem is usually addressed by Kalman Filter (KF) or its nonlinear variants such as Extended Kalman Filter (EKF) and Unscented Kalman Filter (UKF). The SSPE problem, in comparison to the standard state estimation, poses new research challenges such as poor observability. Because unknown or uncertain system parameters should also be estimated along with unknown states by using partial noisy measurements. Due to the high non-linearity involved in SSPE problem, the generic nonlinear filtering approach is usually adopted such as Particle Filtering (PF). PF is a sequential Bayesian approach by Monte Carlo sampling, and is particularly suitable for non-linear and non-Gaussian filtering. In this approach, a large number of particles are drawn to approximate the probability density function of states and parameters (Arulampalam et al., 2002; Zhu et al., 2017; Moradkhani et al., 2005; Li et al., 2015) rather than only propagating the mean and variance. This distribution can provide confidence information which is not possible in point estimation (e.g. optimization based approaches).

It should be noted that in real-life agricultural applications, various types of extra information (or background knowledge) is usually available. For instance, many unknown or uncertain parameters in the AquaCrop model have physical meanings and therefore are with upper and lower bounds. This parameter bound information, if taken into account properly, can further improve the estimation performance of particle filter (Liu et al., 2019; López-Negrete et al., 2011; Amor et al., 2016). It is also discovered in this study that the sensitivity of various parameters in crop models may vary significantly in different crop growth stages, that is, a parameter being sensitive in stage A may become insensitive in stage B and vice versa (Xing et al., 2017). As a result, a static parameter modelling error term is insufficient to capture this dynamic sensitivity behaviour and alternative approaches should be sought. Therefore, this work aims to develop an improved particle filter framework for SSPE of the AquaCrop model, which can accommodate these extra information (including parameter bound information and parameter sensitivity information) for better estimation performance. The improved particle filter is compared against the conventional PF and constrained PF by using both Monte Carlo (MC) simulations and real-life experiment. To be more exact, the main contributions are summarized as follows:

(1) A sequential particle filter with parameter bound and sensitivity information is drawn to integrate AquaCrop model and UAV remote sensing measurements so that the posterior distribution of both states and uncertain parameters can be estimated in near real time.

(2) MC simulations and an experimental case study are performed to validate the developed framework against conventional particle filter and constrained particle filter.

\section{Problems formulation}

The core problem in this study can be formulated as a sequential state and parameter estimation (SSPE) problem by integrating AquaCrop model and remote sensing observations. Since the AquaCrop model is non-analytical, conventional particle filter cannot be used and an open access source AquaCrop model is selected (AquaCrop-OS) (Foster et al., 2017). This model can be modelled as a discrete-time dynamic state- space model, and satisfies a Markov process where the future states at $k+1$ step is only associated with the states at $k$ step (Kanso et al., 2003). The compact system including state dynamics and observation model can be represented by (1).

$\left\{\begin{array}{l}x_{k+1}=F\left(x_{k}, \theta, u_{k}\right)+\nu_{k} \\ Y_{k}=G\left(x_{k}, \theta, u_{k}\right)+\eta_{k}, \text { with } \eta_{k} \sim N(0, \sigma)\end{array}\right.$

where $x_{k+1}$ represents the canopy dynamic states in crop model at $k+1$ time step. $\theta$ and $u_{k}$ denote the selected parameters and forcing data (e.g. weather data, fixed parameters). $Y_{k}$ represents the observations at $k$ time step. $\nu_{k}$ and $\eta_{k}$ are independent, representing the model process noise and measurement noise, which are with zero means and proper covariances, respectively. $F($.$) and G($.$) are non-linear functions relating$ the relevant variables.The SSPE problem in this study is to estimate the state $x_{k}$ and unknown parameter $\theta$ based on the available measurements $Y_{1, \cdots, k}$ up to day $k$.

\section{Methodology}

In this section, some key components of the developed framework are introduced including Sobol sensitive analysis and the improved PF algorithm for SSPE problem of AquaCrop model.

\subsection{Crop parameter sensitivity analysis}

In this section, crop parameter sensitivity analysis is considered. This is because the parameter sensitivity in crop models may vary significantly in different crop growth stages. As a result, new parameter error modelling instead of a static one should be designed to capture the dynamic behaviour of parameter sensitivity.

\subsubsection{Sobol sensitivity analysis}

Sensitive analysis (SA) is one effective tool to quantitatively analyse the uncertain factors (parameters or driving variables) on model outputs and identify the most sensitive ones (Xing et al., 2017). The Sobol method is a variance based approach decomposing the model output variance into contributions associated with each input parameter. For AquaCrop model, it can evaluate the contribution of separate parameters and interactions to the model outputs (e.g. CC or biomass) (Zhang et al., 2015). Crop parameters $c g c, c c x$ and $c d c$ can determine canopy growth gradient, maximum canopy cover and canopy decline gradient at growing stages. An approximate canopy cover growth can be simulated once these three parameters are confirmed. As a consequence, these three parameters are selected for sensitive analysis and estimated in SSPE. Details of Sobol method is referred to Zhang et al. (2015) and Nossent et al. (2011).

\subsection{2. $S A$ results}

The sensitivity analysis results by Sobol analysis are depicted in Fig. 1 including the first order index (left plot) and the total order index (right). It follows from Fig. 1 that both indices share the same sensitivity trend. In particular, $\operatorname{cgc}$ has a high sensitivity during the whole wheat growing period. However, $c c x$ starts its influence when the canopy is saturated; and $c d c$ plays a significant role in crop degrading period. First order sensitive index can be taken into account in the proposed PF framework by adaptively adjusting the parameter modelling error (via variance).

\subsection{PF framework with parameter bound and sensitivity information}

In this section, the improved particle filter based SSPE framework is discussed, which can accommodate the parameter bound and sensitivity information. The SSPE framework for AquaCrop is shown in Fig. 2. 

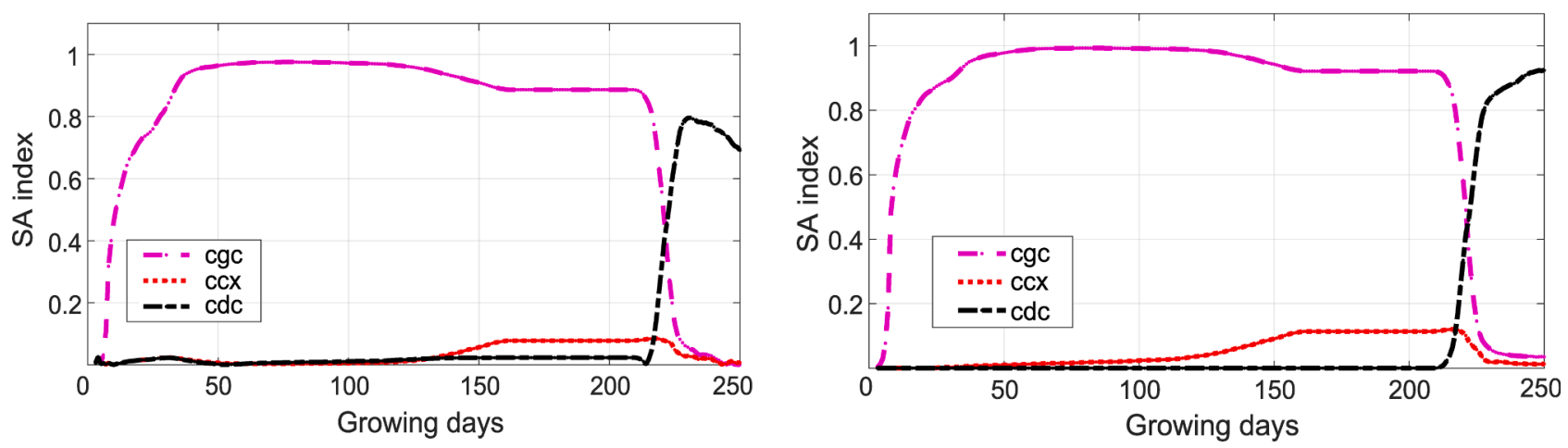

Fig. 1. First order (left) and total order (right) SA index of three parameters over time..

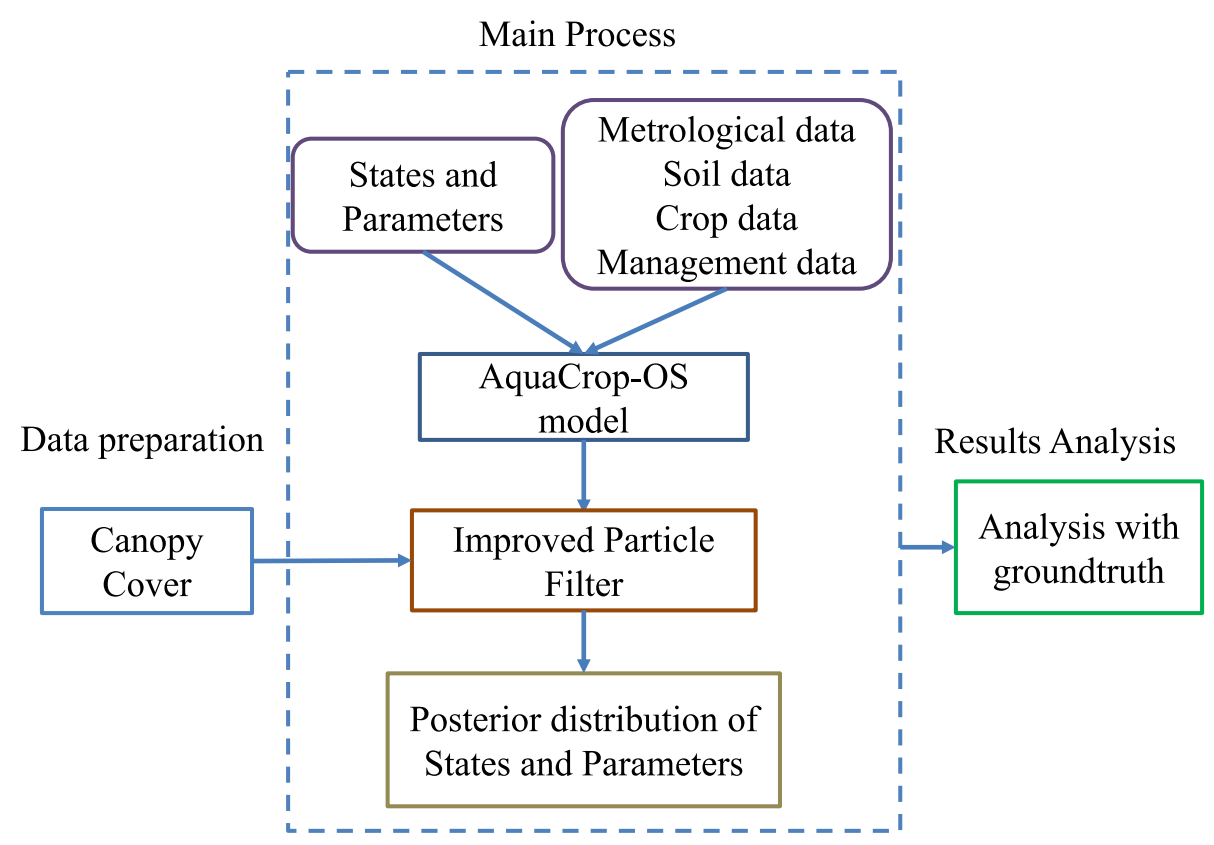

Fig. 2. Framework of the proposed particle filter for state and parameter estimation of the AquaCrop model..

\subsubsection{Recursive AquaCrop model}

As one open-sourced model, the AquaCrop-OS can be easily integrated with different algorithms for various applications (Steduto et al., 2009; Foster et al., 2017; Zhang et al., 2018). However, this crop model need to be revised recursively before realizing its full potentials so that the main relationship between crop states (e.g. biomass, canopy cover) and other properties (e.g. weather, crop and soil parameters, field management) is reformulated using crop water balance principle. Thus, our new program is able to generate and record new crop state in realtime, meanwhile all parameters and states can be easily updated with the advert of new observations. Consequently, this model can complete the crop simulation process by using different filtering algorithms in a recursive way.

\subsubsection{Improved particle filter}

Particle filter, one of recursive filtering methods, can be modified to solve the SSPE problem (Arulampalam et al., 2002). In conventional particle filter, a series of particle samples with corresponding weights are used to model the posterior state distribution (Jiang et al., 2014), where the weight is calculated based on Bayesian equation fusing prior information and observations. However, in some practical scenarios, constrained particle filter is usually utilised as physical principles and process restrictions can be taken into account as constraints (as additional knowledge/information) so that effective particles can be increased to generate a better posterior distribution (Zhao et al., 2012).

In the proposed problem, the parameters to be estimated $\theta_{k}$ are augmented with the original states $x_{k}$ to become the augmented states $X_{k}$,

$X_{k+1}=\left[\begin{array}{l}x_{k+1} \\ \theta_{k+1}\end{array}\right]$

A Gaussian random walk is assumed for the parameters

$\theta_{k+1}=\theta_{k}+\mu_{k}$

where $\mu_{k} \sim N(0, \alpha)$ is a Gaussian distribution with zero mean and a predefined covariance $\alpha$. Consequently, by substituting Eq. (2) and Eq. (3) into Eq. (1), the dynamic model with augmented state $X_{k+1}$ can be rewritten as

$$
\left\{\begin{array}{l}
X_{k+1}=F^{\prime}\left(X_{k}, u_{k}\right)+\nu_{k}^{\prime} \\
Y_{k}=G\left(X_{k}, u_{k}\right)+\eta_{k}
\end{array}\right.
$$

where the modified function $F^{\prime}\left(\right.$.) and modified noise $\nu_{k}^{\prime}$ are given by

$F^{\prime}\left(X_{k}, u_{k}\right)=\left[\begin{array}{c}F\left(x_{k}, \theta_{k}, u_{k}\right) \\ \theta_{k}\end{array}\right], \nu_{k}^{\prime}=\left[\begin{array}{l}\nu_{k} \\ \mu_{k}\end{array}\right]$.

It should be noted that the selection of $\mu_{k}$ (i.e. its variance) is 
paramount for the filtering performance. It follows from Berntorp and Di Cairano (2016) that $\mu_{k}$ reflects the intensity of process noise and the size of sampling range. If a small variance term is chosen, it is difficult to converge to the truth values timely. And if a too large variance is chosen, more invalid particles will be generated, impairing algorithm effectiveness. In order to reduce the adverse effect caused by the inappropriately selected covariance in SSPE problem, the process noise is related to the sensitivity analysis result, which is defined as

$\mu_{k}=H\left(\right.$ index $\left._{k}\right)$

where index $x_{k}$ is time-series parameter sensitive index at day $k$, and $H($. represents the process error function. $H($.$) is designed so that a high$ sensitivity value leads to a smaller error covariance and a low sensitivity value results in a larger error covariance. It will be shown that this strategy can significantly improve the effectiveness of the particle filter.

In real-life agriculture applications, some states and parameters have physical properties and as a result certain constraints information (e.g. bound information) is usually available (Zhao et al., 2012). These constraints can be represented by certain inequality function $g\left(X_{k}\right)<=0$. The probability conditional on $X_{k}$ can be defined as $p\left(D_{k} \mid X_{k}\right)$. In practical implementation, the particles can be accepted if they satisfy the constraints and be rejected if the constraints are violated.

It follows from Liu et al. (2019) that the posterior distribution of $X_{0: k+1}$ with constraints information $D_{1: k+1}$ can be derived according to the Bayesian recursion once measurement $Y_{1: k+1}$ is available, given by

$p\left(X_{0: k+1} \mid Y_{1: k+1}, D_{1: k+1}\right)=\frac{p\left(Y_{k+1} \mid X_{k+1}\right) p\left(D_{k+1} \mid X_{k+1}\right) p\left(X_{k+1} \mid X_{k}\right) p\left(X_{0: k} \mid Y_{1: k}, D_{1: k}\right)}{p\left(Y_{k+1}, D_{k+1} \mid Y_{1: k}, D_{1: k}\right)}$

where $p\left(X_{0: k+1} \mid Y_{1: k+1}, D_{1: k+1}\right)$ describes the posterior distribution at time $k+1$ from the posterior $p\left(X_{0: k} \mid Y_{1: k}, D_{1: k}\right)$ at time $k \cdot p\left(X_{k+1} \mid X_{k}\right)$ denotes the crop model function and $p\left(Y_{k+1} \mid X_{k+1}\right)$ expresses the likelihood function. $p\left(D_{k+1} \mid X_{k+1}\right)$ is hard constraint related probability.

Our proposed $\mathrm{PF}$ applies enough particle samples to approximate the posterior probability density function (PDF), where each particle represents a specific state $X_{k+1}^{i}$ with a proper probability weight $w_{k+1}^{i}$. The posterior PDF of states and parameters could be approximated by $N$ particles and their corresponding weights, given by

$p\left(X_{0: k+1} \mid Y_{1: k+1}, D_{1 ; k+1}\right) \approx \sum_{i=1}^{N} w_{k+1}^{i} \delta\left(X_{0: k+1}-X_{0: k+1}^{i}\right)$

where $N$ means the particle number and $\delta$ is the Dirac delta function. $p\left(X_{0: k+1} \mid Y_{1: k+1}, D_{1 ; k+1}\right)$ is the truth posterior PDF, $X_{0: k+1}^{i}$ is the i-th particle with related weight $w_{k+1}^{i}$.

According to sequential importance sampling principle (particle weight selection), $X_{k+1}$ from proposal distribution $q\left(X_{0: k+1}^{i} \mid Y_{1: K+1}\right.$, $D_{1 ; k+1}$ ) can be generated and assigned with the corresponding weights according to

$w_{k+1}^{i} \propto \frac{p\left(X_{0: k+1}^{i} \mid Y_{1: K+1}, D_{1 ; k+1}\right)}{q\left(X_{0: k+1}^{i} \mid Y_{1: K+1}, D_{1 ; k+1}\right)}$

According to Arulampalam et al. (2002) and De Bernardis et al. (2016), the proposal distribution could be factorised as

$q\left(X_{0: k+1} \mid Y_{1: k+1}, D_{1 ; k+1}\right)=q\left(X_{k+1} \mid X_{0: k}, Y_{1: k+1}, D_{1 ; k+1}\right) q\left(X_{0: k} \mid Y_{1: k}, D_{1 ; k}\right)$

By inputting Eq. (10) and Eq. (7) into the importance weights Eq. (9), particles weights can be updated

$w_{k+1}^{i} \propto \frac{w_{k}^{i} p\left(Y_{k+1} \mid X_{k+1}^{i}\right) p\left(D_{k+1} \mid X_{k+1}^{i}\right) p\left(X_{k+1}^{i} \mid X_{k}^{i}\right)}{q\left(X_{k+1}^{i} \mid X_{0: k}^{i}, Y_{1: k+1}, D_{1 ; k+1}\right)}$

where $q\left(X_{k+1}^{i} \mid X_{0: k}^{i}, Y_{1: k+1}, D_{1 ; k+1}\right)$ means the posterior probability density function affecting the particle filter results. In this study, the proposal distribution is assumed to be the prior information $q\left(X_{k+1} \mid X_{k}, Y_{k+1}, D_{k+1}\right)$ $=p\left(X_{k+1} \mid X_{k}\right)$, thus the above equation can be simplified as

$w_{k+1}^{i} \approx w_{k}^{i} p\left(Y_{k+1} \mid X_{k+1}^{i}\right) p\left(D_{k+1} \mid X_{k+1}^{i}\right)$

Considering the hard constraint property, the constraint probability condition on $X_{k+1}$ can be written as

$p\left(D_{k+1} \mid X_{k+1}^{i}\right)= \begin{cases}1, & \text { if } g\left(X_{k+1}\right)<=0 \\ 0, & \text { otherwise }\end{cases}$

Consequently, the particle weights can be rewritten by considering the constraints information

$w_{k+1}^{i}=\left\{\begin{array}{cl}\propto w_{k}^{i} p\left(Y_{k+1} \mid X_{k+1}^{i}\right), & \text { if } g\left(X_{k+1}\right)<=0 \\ 0, & \text { otherwise }\end{array}\right.$

Assuming that the measurement noise follows a Gaussian distribution with zero mean and a covariance $R$, the likelihood function and updated particle weight are given by Jiang et al. (2014) and Li et al. (2015)

$P\left(Y_{k+1} \mid X_{k+1}^{i}\right)=\frac{1}{\sqrt{2 \pi} \sqrt{R_{k+1}}} \exp \left[-\frac{\left(Y_{k+1}-G\left(X_{k+1}^{i}\right)\right)^{2}}{2 R_{t+1}}\right]$

$w_{k+1}^{i}=\frac{w_{k+1}^{i}}{\sum_{i=1}^{N} w_{k+1}^{i}}$.

Due to the particle degeneracy problem, a useful measure of effective number can be defined as

$N_{\text {eff }}=\frac{1}{\sum_{i=1}^{N}\left(w_{k+1}^{i}\right)^{2}}$.

To attenuate the particle degeneracy problem, resampling strategy is adopted (Li et al., 2015). In particular, Metropolis resampling is employed due to its reliability and a low computation cost. The weight of each particle will be $1 / N$ after resampling process. The posterior distribution of filtered states and parameters is in the form of particles, thus the updated particles after resampling will become the initial particles for the next evolutionary iteration until all available measurements are assimilated into AquaCrop model. The above steps are summarized in Algorithm 1.

Algorithm 1. Particle filter with constraints and sensitivity information

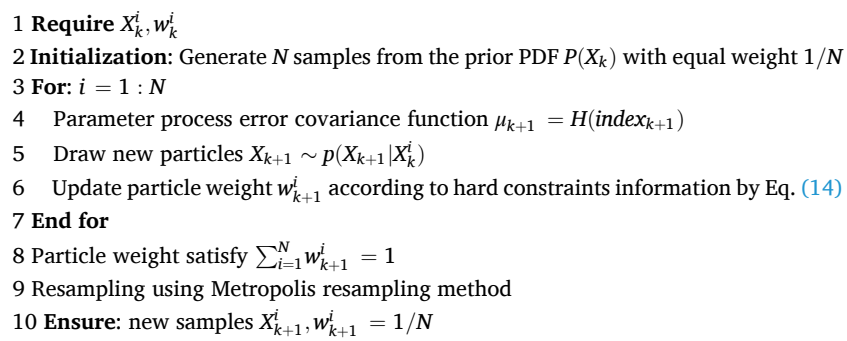

\subsection{Validation method for field experiment}

In this study, two validation methods are adopted for the proposed algorithm including MC simulation in a simulated environment and field validation based on an experimental case study. In particular, due to the lack of groundtruth crop parameter data for field experiment owning to the logistic issues, the validation of field experiment is only based on CC estimation performance instead of both CC and crop parameters estimation. A modified Day Forward-Chaining (DFC) method (Bergmeir and Benítez, 2012; Perotti et al., 2015), as shown in Fig. 3, is used for 


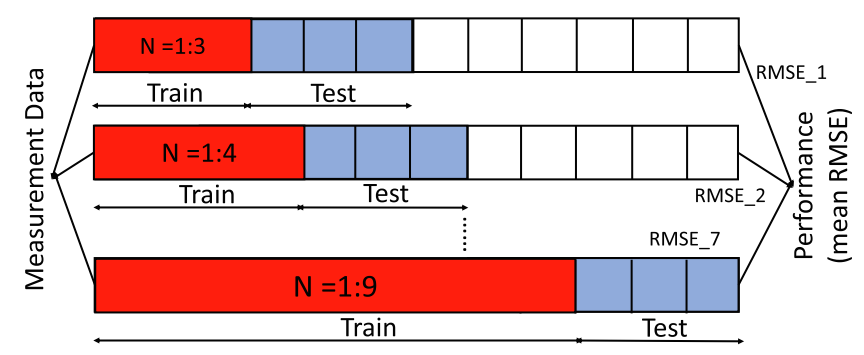

Fig. 3. Day Forward-Chaining validation illustration for field case study.

temporal validation in the field experiment. This is because forward chaining method can avoid the problems that an arbitrary selection of the hold-out test set may lead to a biased estimate, especially for the case with a limited number of observations.

In the modified DFC of Fig. 3, each measurement denotes one fold with 12 folds in total in the field case study. Index $N$ in red cells denotes the first few folds used for training; in other words, the measurements on these first few days are for state and parameter estimation using the proposed particle filter. In real-life agriculture management practice, the decision is usually made by analysing the forecasting information in a number of future dates. Following this line of thought, the three folds following the training folds are used for testing. This means that the estimated state and parameters using the folds in red are inserted into the AquaCrop model to generate the prediction for folds in blue so that performance can be evaluated. The folds used for training and testing are illustrated in Fig. 3.

\section{Systematic settings}

In this section, various validation approaches including Monte Carlo simulation and a real-world experiment case study are implemented to test the improved particle filter against the conventional particle filters with constraints or sensitivity information.

\subsection{Monte Carlo simulation settings}

MC simulations are firstly adopted to evaluate the SSPE performance for AquaCrop model, in particular for parameter estimation. Three parameters strongly associated with dynamic state canopy cover (CC) are selected. Thus, a four dimensional state and parameters vector is defined.

$X=[c g c, c c x, c d c, C C]^{T}$.

The states and parameters with their bound information and definitions are displayed in Table 1. In MC simulation, the default crop parameter values (being constant in its life-cycle) and model-generated $\mathrm{CC}$ in the AquaCrop simulation model are set to be the groundtruth. The noisy observations for model comparisons are derived from groundtruth CC by adding a Gaussian measurement noise with zero mean and a variance of $0.05^{2}$. The time period of the AquaCrop model is consistent with the experiment from 08/Oct/2018 to 06/June/2019 under the same treatment and the measurement interval is 10 days. $50 \mathrm{MC}$ simulations with random initials and random measurement noises are performed to test the robustness of all three methods including the

Table 1

Selected state and parameters definition with bounds information for MC simulation and field experiment.

\begin{tabular}{ccc}
\hline Variables & Prior information & Physical meaning \\
\hline$c g c$ & $(0.005,0.02)$ & Canopy growth coefficient \\
$c c x$ & $(0.82,0.98)$ & Maximum canopy cover fraction \\
$c d c$ & $(0,0.02)$ & Canopy decline coefficient \\
$c C$ & $(0,1)$ & Canopy cover \\
\hline
\end{tabular}

conventional PF, the PF with constraints and the improved PF with constraints and sensitivity information.

\subsection{Experimental evaluation}

In addition to MC simulation, experimental verification is also performed. There is one case study (winter wheat) conducted from 2018 to 2019 to validate the proposed method. The key model state, wheat canopy cover, is extracted from multi-spectral images as below.

\subsubsection{Experiment wheat field and UAV aerial imaging}

The experiment site is located at Caoxinzhuang experiment field, which belongs to Northwest Agriculture and Forestry University (see Fig. 4 for the location) (Su et al., 2018). The climate in this area is characterized by semi-humidity and semi-aridity, with an average annual temperature of $12.9^{\circ} \mathrm{C}$. In this study, the cultivar Xiaoyan22 (winter wheat) is adopted, which was developed by Northwest A\&F university. In addition, some key information that is required in AquaCrop model, such as meteorological data and basic soil data can be downloaded from National Meteorological Information Center (http://data.cma.cn) and national Earth system Science Data Sharing Infrastructure (http://www.geodata.cn).

In this study, UAV remote sensing images are preferred due to its high spatial/spectral resolutions. In particular, commercial DJI M100 Quadrotor (DJI Company, Shenzhen, China) and RedEdge camera (MicaSense Company, Seattle, USA) with five multi-spectral bands was integrated as the UAV sensing system (see Fig. 5). RedEdge camera, in comparison to conventional RGB camera, has extra Rededge and NIR bands, providing extra spectral information for better classification performance (Su et al., 2019). The weight, dimensions and image resolution of RedEdge camera are $135 \mathrm{~g}, 5.9 \mathrm{~cm} \times 4.1 \mathrm{~cm} \times 3.0 \mathrm{~cm}$ and $1280 \times 960$ pixels, respectively.

In each flight, the RedEdge camera was fixed on the UAV, pointing vertically downwards to the wheat canopy. Flight altitude was set to be about 20 meters above ground with a ground image spatial resolution of about $1.2 \mathrm{~cm} /$ pixel. Image overlap and sidelap were set to be up to $75 \%$ for an accurate orthomosaic generation. Reflectance calibration panel was always imaged at $1 \mathrm{~m}$ height before each flight to account for camera and reflectance characteristics, and environmental variations (Zhang et al., 2019; Su et al., 2018). After data collection, Pix4DMapper, a commercial photogrammetry software, was then used to process the raw images to generate the caliberated orthomosaic images for each band. The overall process includes initial processing, Point Cloud and Mesh generation and orthomosaic generation, where more details are referred to the existing studies (Su et al., 2018, 2019). Finally, a total of twelve multi-spectral images were collected covering winter wheat key developmental stages (please refer to Table 2 for the specific imaging times) including tillering stage, green-up stage, jointing stage, anthesis stage and grain filling stage (Ozturk et al., 2004).

\subsubsection{CC calculation}

In this study, the CC calculation can formulated as a wheat/nonwheat two-class classification problem so that the wheat pixel proportion can be calculated for the region of interest. The overall process is displayed in Fig. 6, which include several components such as data labelling, random forest classifier and CC value calculation. One typical example for the data on $16 / \mathrm{Dec} / 2018$ is presented, where these steps are detailed in the following subsections.

Supervised classification depends on data labelling. In this study, wheat and non-wheat pixels are directly labelled according to on-site experiment and UAV RGB color image, where the RGB image is generated by using Red-Green-Blue bands of the multispectral image. The labelled sample image is displayed in Fig. 7, where wheat pixels (wheat), non-wheat (others) and unlabelled pixels (un) are represented in different colours. Moreover, all available five bands including Blue (B), Green (G), Red (R), RedEdge and Near-infrared (NIR) bands are selected 


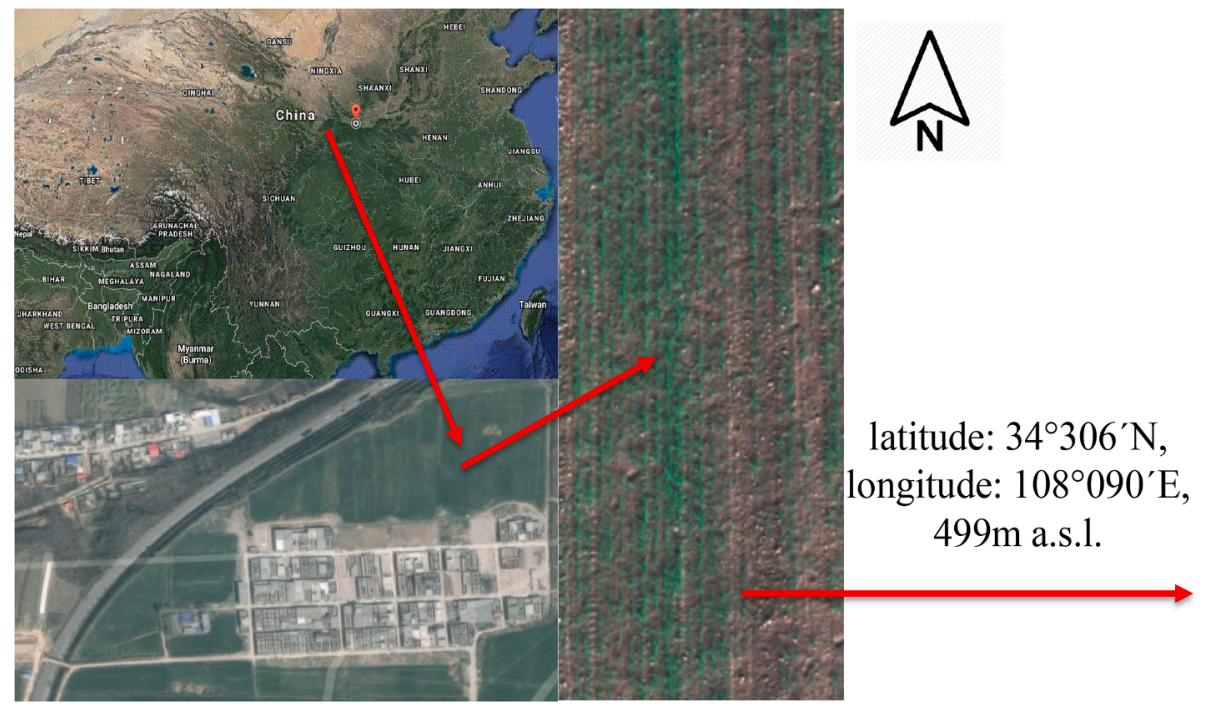

Fig. 4. Geographic details of the study area.

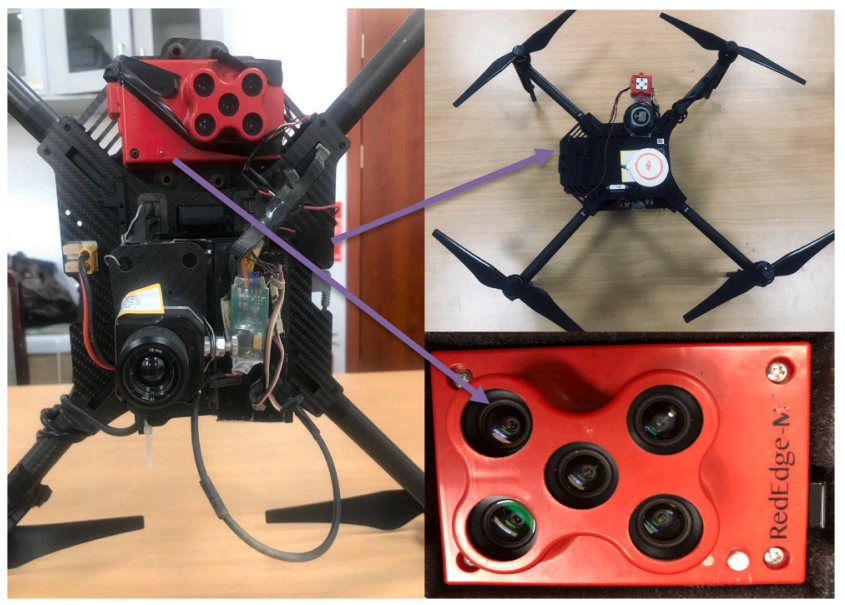

Fig. 5. UAV camera system: DJI M100 Quadrotor UAV (left), GPS (upper right) and RedEdge camera (lower right).

Table 2

Canopy cover values over time.

\begin{tabular}{cccc}
\hline Date & CC Value & Date & CC Value \\
\hline $11 / 11 / 2018$ & 0.362 & $09 / 12 / 2018$ & 0.7972 \\
$16 / 12 / 2018$ & 0.8296 & $30 / 12 / 2018$ & 0.8973 \\
$03 / 03 / 2019$ & 0.9361 & $25 / 03 / 2019$ & 0.9494 \\
$30 / 03 / 2019$ & 0.9649 & $05 / 04 / 2019$ & 0.9874 \\
$15 / 04 / 2019$ & 0.9775 & $23 / 04 / 2019$ & 0.9893 \\
$27 / 04 / 2019$ & 0.9686 & $02 / 05 / 2019$ & 0.9321 \\
\hline
\end{tabular}

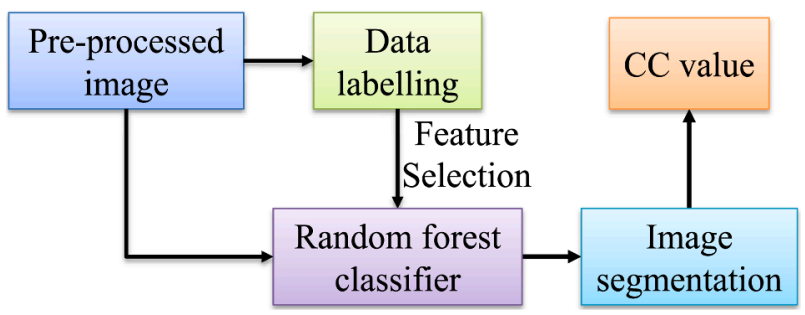

Fig. 6. Steps for canopy cover calculation by using remote sensing images and random forest classifier. as the features for supervised classification. Spectral comparison between wheat and non-wheat pixels is referred to Zhang et al. (2019).

A classifier is then required to perform the classification task so that new aerial images can be automatically classified for CC calculations. In this study, random forest classifier is implemented due to its good performance in terms of accuracy and robustness and a relatively low computation load, where the hyper-parameters are further automatically tuned by using Bayesian optimization (Su et al., 2018). Random forest algorithm has been previously used for wheat canopy segmentation in previous studies (Zhang et al., 2019), which show that an accuracy of $99 \%$ can be achieved in selected labelled dataset. The detailed algorithm is omitted due to the lack of space and is referred to Su et al. (2018) and Thornton et al. (2013).

The labelled image is split into training and testing data with a proportion of $70 \%$ and $30 \%$. The trained random forest classifier is then applied to the original example image, where the classification map is displayed in the right plot of Fig. 7. Then CC value can be calculated by $C C=N_{w p} /\left(N_{w p}+N_{n w p}\right)$, where $N_{w p}$ and $N_{n w p}$ represent the number of wheat and non-wheat pixels in the region of interest. All CC values over time are displayed in Table 2 by following the above steps.

\subsubsection{Experiment settings}

Measurement noise of the experiment data can be estimated by using the algorithm in Zhang et al. (2019), where the covariance value is set to be 0.0021 . The prior information of state and parameters as well as other settings of the improved PF algorithm remain the same as MC simulation (see Table 1).

\section{Results and discussion}

This section demonstrates a comparative estimation result using various PF methods. For MC simulation, the SSPE performance is evaluated by the root mean squared error (RMSE) of all MC runs. While in experimental validation, due to the absence of parameters groundtruth, error analysis is only tested on CC.

\subsection{Results of $M C$ simulation}

MC analysis with random initial values and various measurements is first performed for the three methods. Mean and variance can be calculated from all particles at each observation day for one MC simulation run. For RMSE comparison, the estimated parameters on the last day of each MC run are used to calculate the RMSE value. In addition to 


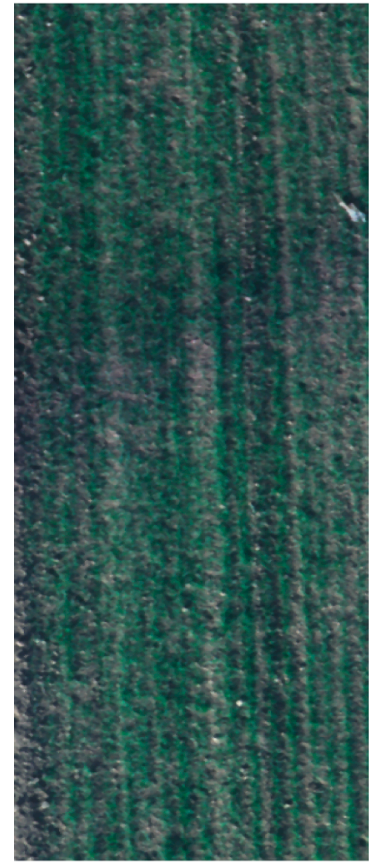

A

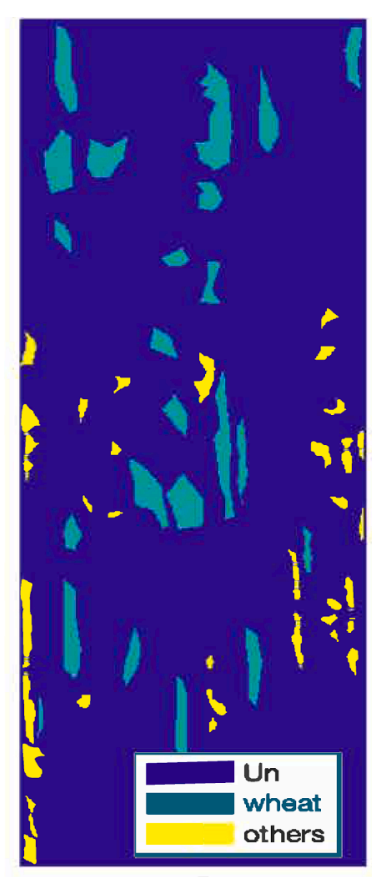

B

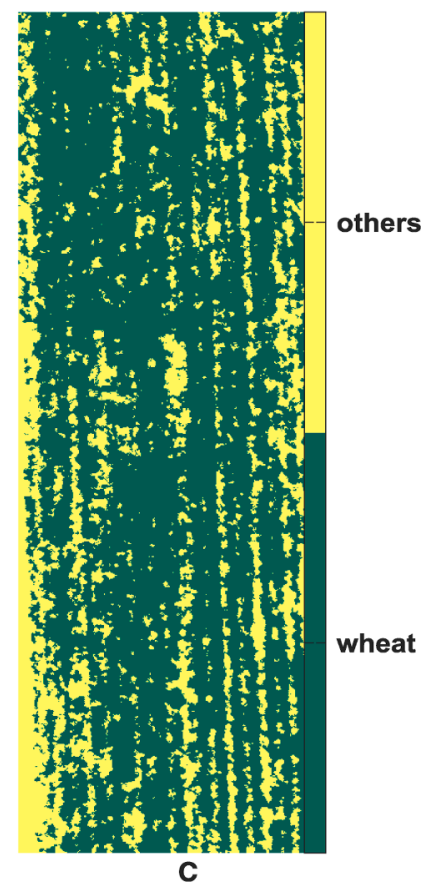

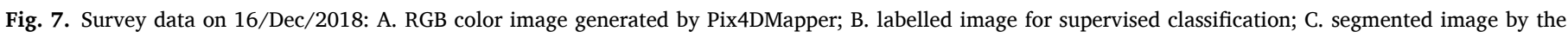
random forest classifier. (For interpretation of the references to colour in this figure legend, the reader is referred to the web version of this article.)

parameter comparison, for CC, the total error is obtained by using the mean RMSE of all MC runs, where the RMSE of each run can be calculated by all CC estimations against the groundtruth at all observation dates. In particular, in MC simulations, all parameters to be estimated are constant in one certain local field and the default parameters are set to be groundtruth for performance evaluation (Steduto et al., 2009).

The RMSE value of each method is shown in Table 3. Meanwhile, the error is also displayed, where ' ${ }^{\prime}$ means the error reduction and ' + ' denotes the error increment in comparison to RMSE value using conventional PF. The RMSE error using different methods is defined by the following formula.

$E_{P P F}=\frac{R M S E_{P P F}-R M S E_{P F}}{R M S E_{P F}} * 100 \% ; E_{C P F}=\frac{R M S E_{C P F}-R M S E_{P F}}{R M S E_{P F}} * 100 \%$

where $E_{P P F}$ and $E_{C P F}$ denote the parameters error using the proposed PF and constrained $\mathrm{PF}$ in comparison to using conventional PF. RMSE $P P F$, $R M S E_{C P F}$ and $R M S E_{P F}$ represent the RMSE of estimated parameters and state CC using the proposed method, constrained PF and conventional $\mathrm{PF}$, respectively.

It follows from Table 3 that the RMSE value of the proposed method is much smaller than the constrained PF and the conventional PF in terms of all parameters and canopy cover over $50 \mathrm{MC}$ simulations. For cdc estimation, the result is not as good as other parameter estimation, the possible reason is that, as shown in Fig. 1, CC is not sensitive to cdc parameter for most of the growing period. However, one can still see that the proposed PF and the constrained PF result in $16.4 \%$ and $5.2 \%$ improvement over the conventional PF in terms of canopy cover RMSE, which is significant in error percentage. The relationship between the

Table 3

RMSE of 50 Monte Carlo simulations using different particle filter methods.

\begin{tabular}{cccc}
\hline Parameters & Proposed PF(error) & Constrained PF(error) & Conventional PF \\
\hline$c g c$ & $0.000592(-60.7 \%)$ & $0.000833(-44.6 \%)$ & 0.001506 \\
$c d c$ & $0.010725(-31.5 \%)$ & $0.035224(+125 \%)$ & 0.015655 \\
$c c x$ & $0.002034(-71.8 \%)$ & $0.008303(+15 \%)$ & 0.007217 \\
$C C$ & $0.014137(-16.4 \%)$ & $0.016027(-5.2 \%)$ & 0.016907 \\
\hline
\end{tabular}

parameters cdc, cgc, ccx and CC is complex and generally nonlinear, and the weightings of different parameters on $\mathrm{CC}$ are also diverse and timevarying (since the parameter sensitivity is time-varying). As a result, the CC estimation performance may improve even some parameters estimation being poorer (by comparing the constrained $\mathrm{PF}$ and conventional $\mathrm{PF}$ ). But our proposed PF still results in (significantly) better performance over the conventional PF for both CC and model parameters estimation. Consequently, it can be summarised that the proposed method can improve the SSPE estimation performance on both parameter and canopy cover whereas the constrained PF can only marginally improve the estimation performance on $c g c$ and canopy cover.

An error bar of one MC simulation is given in Figs. 8-10 to evaluate the estimation performance by uncertainty analysis, where mean value and self-defined 1.5 times the standard deviation of all generated particles at each observation day are in blue, and the red line and points denote the groundtruth parameter and CC, respectively. It can be seen from Fig. 10 that all estimated parameters are close to the groundtruth after sensitive period. In contrast, some parameters do not converge well by using other PF methods due to the absence of constraints and sensitivity information. Furthermore, it can also be seen from $C C$ results that all estimated CC are all closer to the truth CC by using the proposed PF. In addition to mean value, it can be visually seen that the uncertainties by the proposed method are the smallest among these three methods, mainly due to the sensitivity information making the measurements more efficient in the process of estimation. Therefore, the proposed method achieves the best performance on parameter and CC estimation in terms of stability and accuracy.

In addition, the time-series 3D histogram of state and parameter estimation distribution of one MC run is also displayed in Fig. 11. It can be seen that PF can provide posterior distribution of each parameter and state instead of point estimation, and therefore it can provide estimation confidence. The confidence rule is that the less spread the distribution is, the more reliable the estimation is. As is shown in Fig. 11.C, the state and parameters distribution with small variance can take effects on sensitive period and thereafter. Consequently, in comparison to the conventional $\mathrm{PF}$ and constrained PF, the proposed PF with both constraints and sensitivity information achieve the best estimation with concentrated 

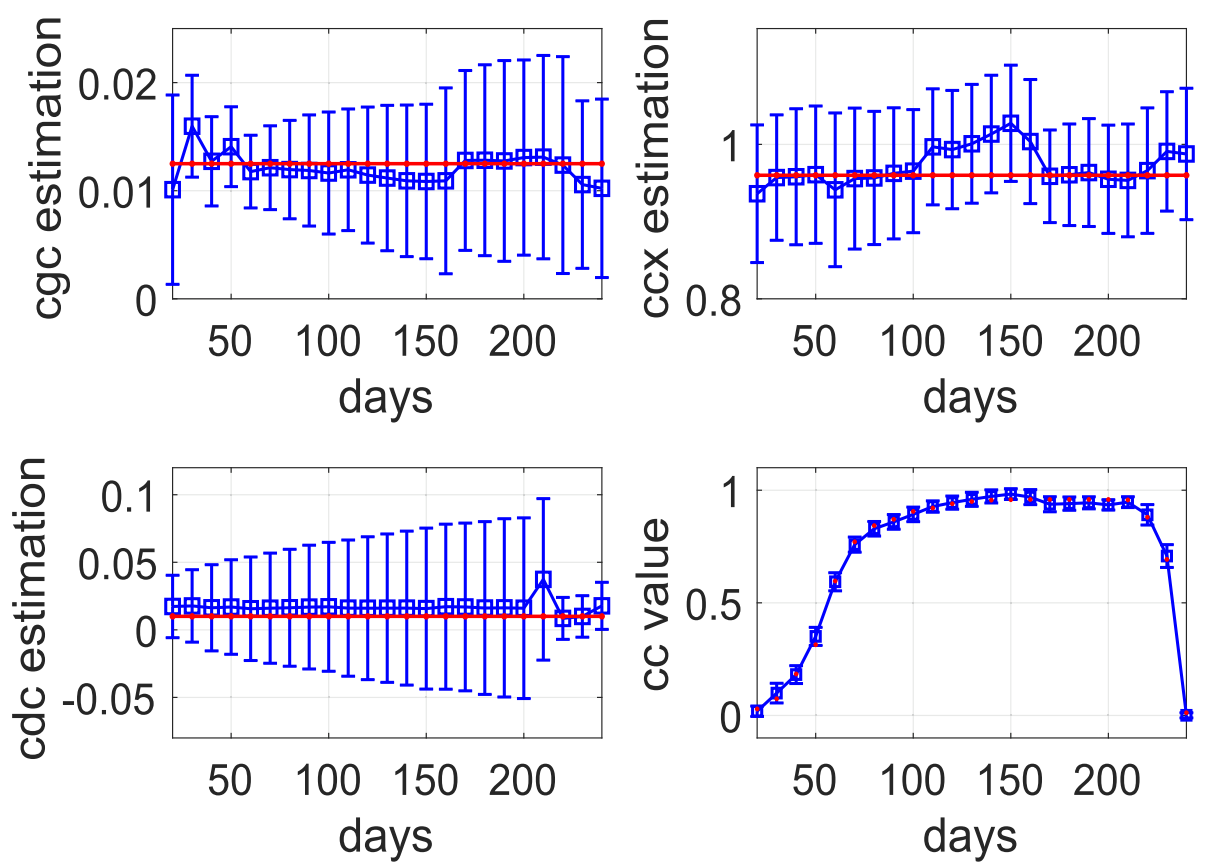

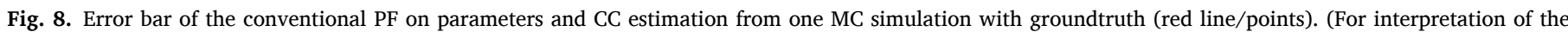
references to colour in this figure legend, the reader is referred to the web version of this article.)
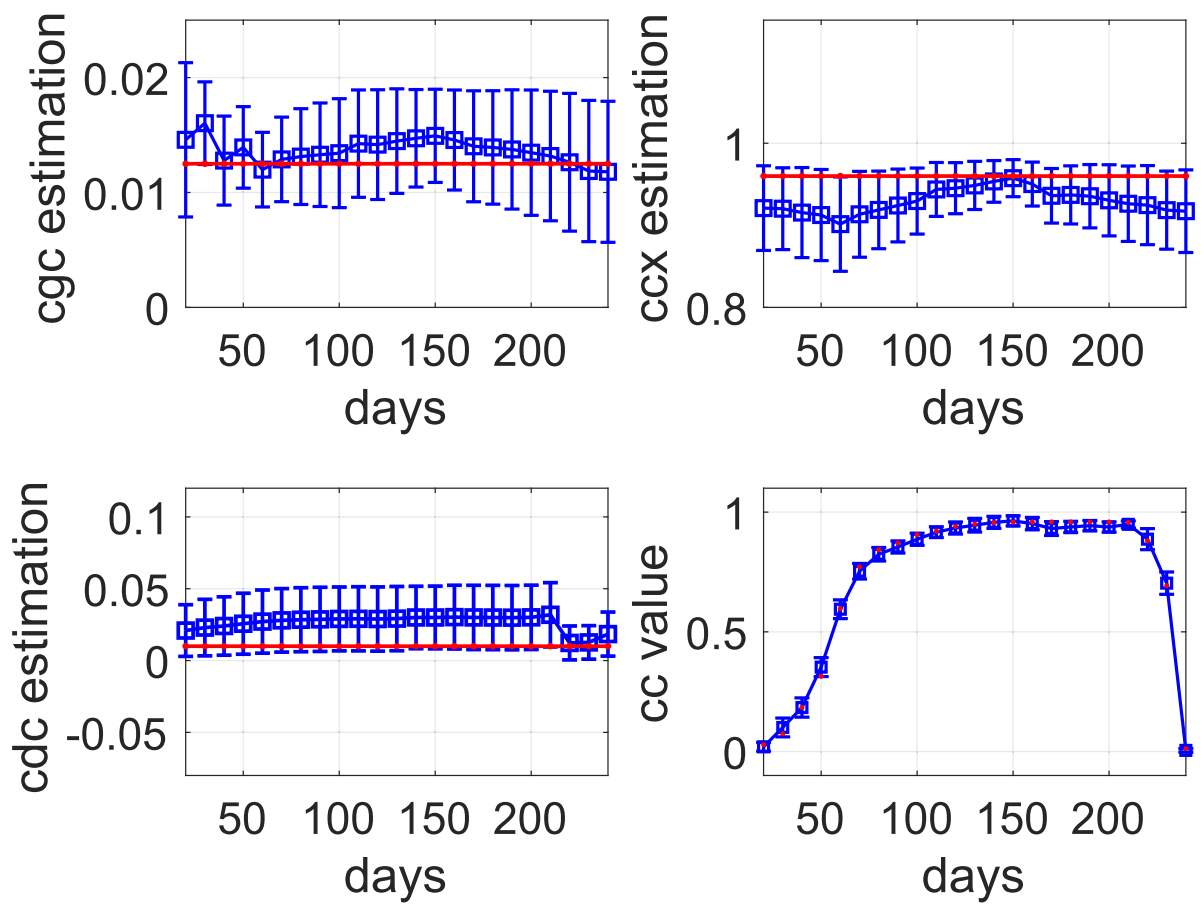

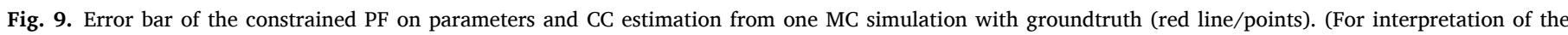
references to colour in this figure legend, the reader is referred to the web version of this article.)

distributions.

\subsection{Results of experimental validation}

By using the DFC validation method, the first few folds are used for training and the following three ones are for testing. Testing data at each run derives a RMSE value against canopy cover observation value. The final validation performance is evaluated in terms of mean RMSE of all runs to test the algorithm robustness. In addition to the aforementioned three SSPE approaches, the default parameter-based canopy cover is also simulated for the field experiment using the default parameter values (cgc $=0.0111 ; \operatorname{ccx}=0.9051 ; \operatorname{cdc}=0.0300)$ and noisy CC. The comparative results with error percentage against conventional PF are summarized in Table 4. It follows from Table 4 that: (1) all SSPE approaches significantly outperform the default parameter based one (i.e. without parameter estimation); (2) our proposed method achieves the smallest RMSE among these four methods.

It follows that the experimental validation performance is not as 

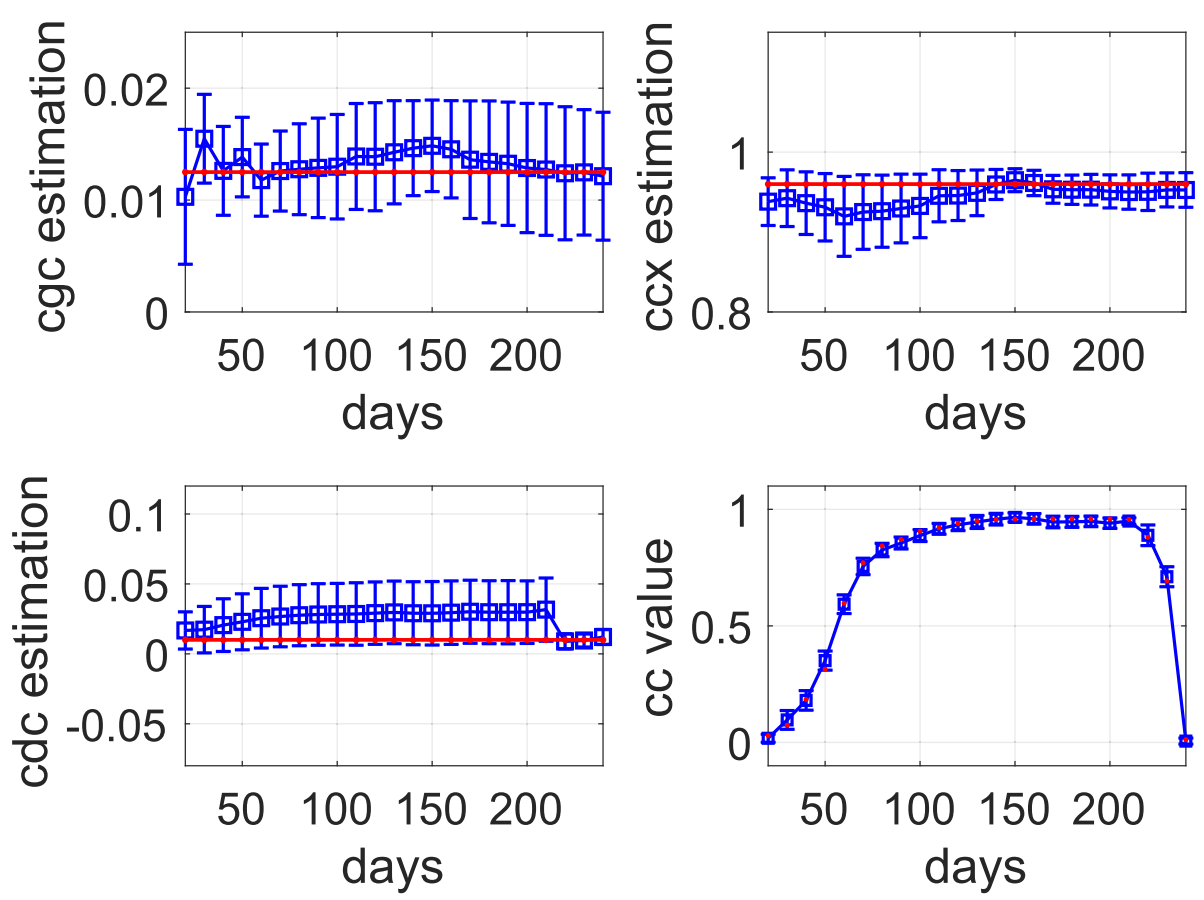

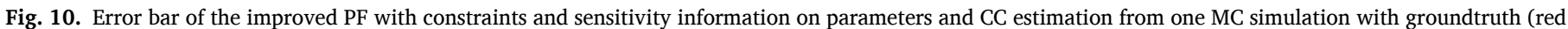
line/points). (For interpretation of the references to colour in this figure legend, the reader is referred to the web version of this article.)
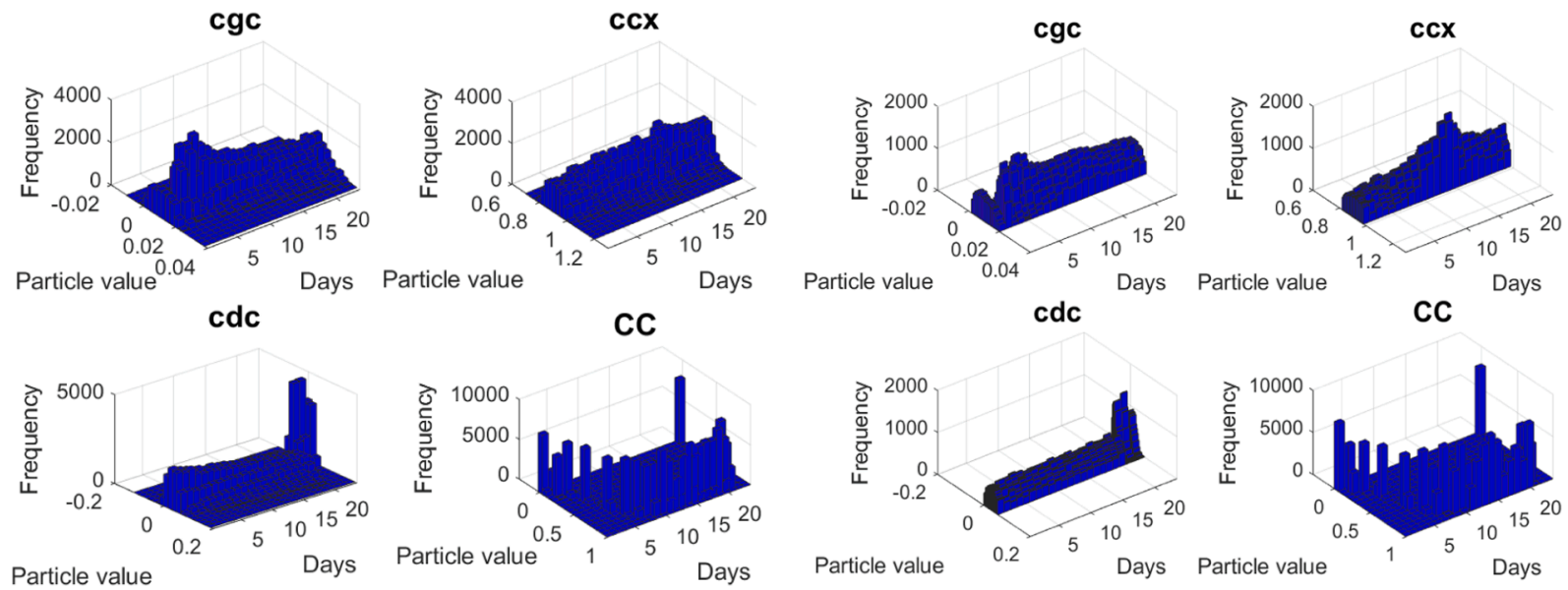

A
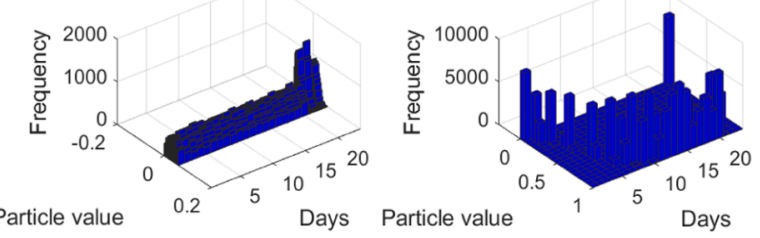

B

cgc
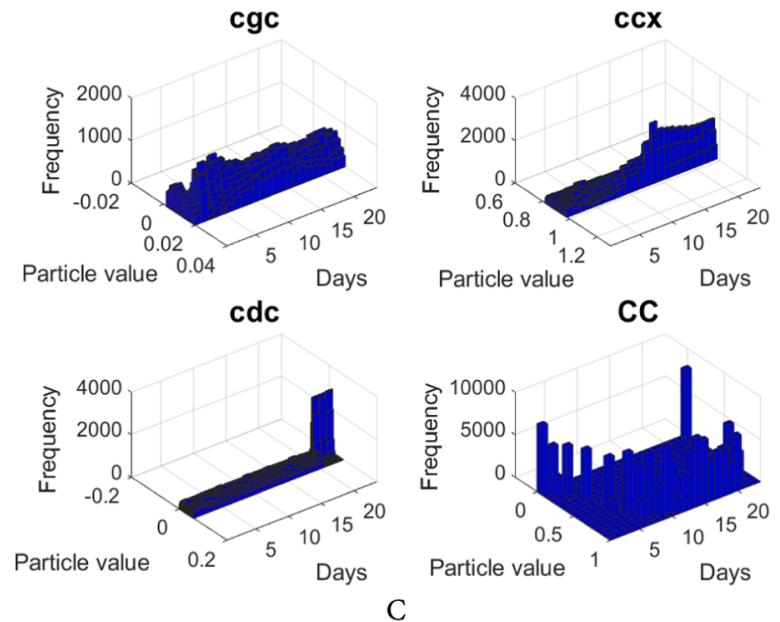

Fig. 11. Time-series 3D histogram of one MC run by different methods: A. conventional PF; B. constrained PF; C. proposed PF. 
Table 4

Mean RMSE of different methods by DFC validation.

\begin{tabular}{ccccc}
\hline State & $\begin{array}{c}\text { Proposed PF } \\
\text { (error) }\end{array}$ & $\begin{array}{c}\text { Constrained PF } \\
\text { (error) }\end{array}$ & $\begin{array}{c}\text { Conventional } \\
\text { PF }\end{array}$ & $\begin{array}{c}\text { Default Parameter } \\
\text { PF(error) }\end{array}$ \\
\hline CC & $\begin{array}{c}0.0656 \\
(-4.8 \%)\end{array}$ & $0.0691(+0.2 \%)$ & 0.0689 & $0.0758(+10.0 \%)$ \\
& & & & \\
\hline
\end{tabular}

good as MC simulation among three methods. It may be due to the lack of measurements at certain key sensitive stages. Meanwhile, the performance of constrained PF is worse than the conventional PF. The possible reason is that the constrained $\mathrm{PF}$ reduces the number of particles and the measurement is not enough to get a better estimation on CC value. Still it can be concluded that all three methods with parameter estimation are capable of solving the SSPE problem for the AquaCrop model, however, the estimation performance of the proposed method considering both constraints and sensitivity information is the best.

\section{Conclusions and future work}

This paper introduces an improved particle filter framework to integrate UAV multispectral images into AquaCrop model so that state and parameter estimation performance can be improved for the AquaCrop model. Machine learning classifier is applied to UAV multispectral image to calculate canopy cover value for winter wheat. Then particle filter is drawn to assimilate canopy cover information and AquaCrop model information in deriving posterior distributions for state and parameters. Notably, crop sensitivity information is accommodated in the improved particle filter in addition to model parameter bound information. Both Monte Carlo simulations and experimental case study are conducted to assess the performance of the improved particle filter against conventional and constrained particle filter. Monte Carlo simulation shows that the proposed method yields the best performance on state and parameter estimation. The proposed approach also obtains accurate canopy cover estimation in experiment in term of root mean square error. Consequently, the proposed approach provides one alternative to the existing particle filter methods for the simultaneous state and parameter estimation problem.

The AquaCrop model is a very useful model for crop management (e. g. crop growth monitoring, irrigation decision). However, some key crop model parameters should be estimated for local farmlands in order to accurately reflect the local behaviour so that its full potentials can be realized. This study achieves this objective by developing a state and parameter estimation algorithm by using particle filter along with parameter sensitivity information. The developed algorithm generally outperforms the conventional approaches, and therefore results in better simulation performance. As a result, this study provides a better management model to the local farmers, so that they can manage their fields in a more precise and sustainable manner. Therefore, local farmers can potentially benefit from an increased productivity while with a reduced input (e.g. water resources). Although the results are promising, there is still much room for further improvement, the number of observations need to be increased and obtained at crop sensitive stage in real-world experiment; in addition, more state information (e.g. biomass, yield) can be collected to further evaluate the algorithm performance.

\section{CRediT authorship contribution statement}

Tianxiang Zhang: Investigation, Software, Visualization, Writing original draft. Jinya Su: Data curation, Methodology, Writing - review \& editing. Cunjia Liu: Conceptualization, Methodology, Supervision, Writing - review \& editing. Wen-Hua Chen: Supervision, Writing - review \& editing, Funding acquisition.

\section{Declaration of Competing Interest}

The authors declare that they have no known competing financial interests or personal relationships that could have appeared to influence the work reported in this paper.

\section{Acknowledgements}

This work was supported by Science and Technology Facilities Council (STFC) under Newton fund with Grant No. ST/N006852/1.

\section{References}

Amor, N., Bouaynaya, N., Georgieva, P., Shterenberg, R., Chebbi, S., 2016. Eeg dynamic source localization using constrained particle filtering. In: 2016 IEEE Symposium Series on Computational Intelligence (SSCI). IEEE, pp. 1-8.

Arulampalam, M.S., Maskell, S., Gordon, N., Clapp, T., 2002. A tutorial on particle filters for online nonlinear/non-gaussian bayesian tracking. IEEE Trans. Signal Process. 50 (2), 174-188.

Bansod, B., Singh, R., Thakur, R., Singhal, G., 2017. A comparision between satellite based and drone based remote sensing technology to achieve sustainable development: a review. J. Agric. Environ. Int. Develop. (JAEID) 111 (2), 383-407.

Bergmeir, C., Benítez, J.M., 2012. On the use of cross-validation for time series predictor evaluation. Inf. Sci. 191, 192-213.

Berntorp, K., Di Cairano, S., 2016. Process-noise adaptive particle filtering with dependent process and measurement noise. In: 2016 IEEE 55th Conference on Decision and Control (CDC). IEEE, pp. 5434-5439.

De Bernardis, C., Vicente-Guijalba, F., Martinez-Marin, T., Lopez-Sanchez, J., 2016. Particle filter approach for real-time estimation of crop phenological states using time series of ndvi images. Remote Sens. 8 (7), 610.

Fiala, A.C., Garman, S.L., Gray, A.N., 2006. Comparison of five canopy cover estimation techniques in the western oregon cascades. Forest Ecol. Manage. 232 (1-3), 188-197.

Foster, T., Brozović, N., Butler, A., Neale, C., Raes, D., Steduto, P., Fereres, E., Hsiao, T.C., 2017. Aquacrop-os: an open source version of fao's crop water productivity model. Agric. Water Manage. 181, 18-22.

Hamuda, E., Glavin, M., Jones, E., 2016. A survey of image processing techniques for plant extraction and segmentation in the field. Comput. Electron. Agric. 125, 184-199.

Hutchinson, M., Liu, C., Chen, W.-H., 2019. Source term estimation of a hazardous airborne release using an unmanned aerial vehicle. J. Field Robot. 36 (4), 797-817.

Jiang, Z., Chen, Z., Chen, J., Liu, J., Ren, J., Li, Z., Sun, L., Li, H., 2014. Application of crop model data assimilation with a particle filter for estimating regional winter wheat yields. IEEE J. Sel. Top. Appl. Earth Observ. Remote Sens. 7 (11), 4422-4431.

Jin, X., Kumar, L., Li, Z., Feng, H., Xu, X., Yang, G., Wang, J., 2018. A review of data assimilation of remote sensing and crop models. Eur. J. Agron. 92, 141-152.

Kanso, A., Gromaire, M.-C., Gaume, E., Tassin, B., Chebbo, G., 2003. Bayesian approach for the calibration of models: application to an urban stormwater pollution model. Water Sci. Technol. 47 (4), 77-84.

Li, H., Chen, Z., Wu, W., Jiang, Z., Liu, B., Hasi, T., 2015. Crop model data assimilation with particle filter for yield prediction using leaf area index of different temporal scales. In: 2015 Fourth International Conference on Agro-Geoinformatics (Agrogeoinformatics). IEEE, pp. 401-406.

Li, T., Bolic, M., Djuric, P.M., 2015. Resampling methods for particle filtering: classification, implementation, and strategies. IEEE Signal Process. Mag. 32 (3), $70-86$.

Liu, C., Li, B., Chen, W.-H., 2019. Particle filtering with soft state constraints for target tracking. IEEE Trans. Aerosp. Electron. Syst. 55 (6), 3492-3504.

López-Negrete, R., Patwardhan, S.C., Biegler, L.T., 2011. Constrained particle filter approach to approximate the arrival cost in moving horizon estimation. J. Process Control 21 (6), 909-919.

Moradkhani, H., Hsu, K.-L., Gupta, H., Sorooshian, S., 2005. Uncertainty assessment of hydrologic model states and parameters: sequential data assimilation using the particle filter. Water Resour. Res. 41 (5).

Nossent, J., Elsen, P., Bauwens, W., 2011. Sobol'sensitivity analysis of a complex environmental model. Environ. Modell. Softw. 26 (12), 1515-1525.

Ozturk, A., Unlukara, A., Ipek, A., Gurbuz, B., 2004. Effects of salt stress and water deficit on plant growth and essential oil content of lemon balm (melissa officinalis 1.). Pak. J. Bot. 36 (4), 787-792.

Perotti, A., Boella, G., Garcez, A.d., 2015. Runtime verification through forward chaining. In: Runtime Verification. Springer, pp. 185-200.

Steduto, P., Hsiao, T.C., Raes, D., Fereres, E., 2009. Aquacrop-the fao crop model to simulate yield response to water: I. Concepts and underlying principles. Agron. J. 101 (3), 426-437.

Su, J., Liu, C., Coombes, M., Hu, X., Wang, C., Xu, X., Li, Q., Guo, L., Chen, W.-H., 2018. Wheat yellow rust monitoring by learning from multispectral uav aerial imagery. Comput. Electron. Agric. 155, 157-166.

Su, J., Liu, C., Hu, X., Xu, X., Guo, L., Chen, W.-H., 2019. Spatio-temporal monitoring of wheat yellow rust using uav multispectral imagery. Comput. Electro. Agric. 167, 105035. 
Su, J., Yi, D., Su, B., Mi, Z., Liu, C., Hu, X., Xu, X., Guo, L., Chen, W.-H., 2020. Aerial visual perception in smart farming: field study of wheat yellow rust monitoring. IEEE Trans. Industr. Inf.

Su, J., Coombes, M., Liu, C., Zhu, Y., Song, X., Fang, S., Guo, L., Chen, W.-H., 2020. Machine learning-based crop drought mapping system by uav remote sensing rgb imagery. Unmanned Syst. 8 (01), 71-83.

Thornton, C., Hutter, F., Hoos, H.H., Leyton-Brown, K., 2013. Auto-weka: Combined selection and hyperparameter optimization of classification algorithms. In: Proceedings of the 19th ACM SIGKDD International Conference on Knowledge Discovery and Data Mining. ACM, pp. 847-855.

Xing, H., Xu, X., Li, Z., Chen, Y., Feng, H., Yang, G., Chen, Z., 2017. Global sensitivity analysis of the aquacrop model for winter wheat under different water treatments based on the extended fourier amplitude sensitivity test. J. Integr. Agric. 16 (11), 2444-2458.
Zhang, X.-Y., Trame, M., Lesko, L., Schmidt, S., 2015. Sobol sensitivity analysis: a tool to guide the development and evaluation of systems pharmacology models. CPT: Pharmacometrics Syst. Pharmacol. 4 (2), 69-79.

Zhang, T., Su, J., Liu, C., Chen, W.-H., 2018. Bayesian calibration of aquacrop model. In: Chinese Control Conference, the 37th, IEEE.

Zhang, T., Su, J., Liu, C., Chen, W.-H., 2019. Bayesian calibration of aquacrop model for winter wheat by assimilating uav multi-spectral images. Comput. Electron. Agric. $167,105052$.

Zhao, Z., Huang, B., Liu, F., 2012. A particle filter based on a constrained sampling method for state estimation. In: 2012 15th International Conference on Information Fusion. IEEE, pp. 816-823.

Zhu, Z., Meng, Z., Cao, T., Zhang, Z., Dai, Y., 2017. Particle filter-based robust state and parameter estimation for nonlinear process systems with variable parameters. Meas. Sci. Technol. 28 (6), 065003. 